Revue d'histoire de l'Amérique française

REVUE D.HISTOIRE DE L'AMÉRIQUE FRANÇAISE

\title{
Doit-on mêler histoire et politique ?
}

Réponse à une critique de M. Jacques Gouin sur un livre de M.

Robert-Lionel Séguin, La Victoire de Saint-Denis, éd. " Parti

Pris ", 1968 ; voir RHAF, XXII : 122-124

\section{Robert-Lionel Séguin}

Volume 22, numéro 2, septembre 1968

URI : https://id.erudit.org/iderudit/302790ar

DOI : https://doi.org/10.7202/302790ar

Aller au sommaire du numéro

Éditeur(s)

Institut d'histoire de l'Amérique française

ISSN

0035-2357 (imprimé)

1492-1383 (numérique)

Découvrir la revue

Citer ce document

Séguin, R.-L. (1968). Doit-on mêler histoire et politique ? Réponse à une critique de M. Jacques Gouin sur un livre de M. Robert-Lionel Séguin, La Victoire de Saint-Denis, éd. " Parti Pris ", 1968 ; voir RHAF, XXII : 122-124. Revue d'histoire de l'Amérique française, 22(2), 319-320. https://doi.org/10.7202/302790ar d'utilisation que vous pouvez consulter en ligne. 
(Réponse à une critique de M. Jacques Gouin sur un livre de M. Robert-Lionel Séguin, La Victoire de Saint-Denis, éd. "Parti Pris", 1968; voir RHAF., XXII : 122-124).

\section{DOIT-ON MELER HISTOIRE ET POLITIQUE ?}

Un auteur n'a pas à pleurnicher si la critique ne lui est pas entièrement favorable. C'est la règle du jeu. Monsieur Jacques Gouin peut-il invoquer cette même règle après son appréciation de La Victoire de Saint-Denis? Qu'il soit permis d'en douter. C'est que monsieur Gouin n'a pas jugé l'œuvre précitée en fonction de l'histoire mais plutôt de la politique.

Monsieur Gouin s'alarme du fait que cette monographie se termine ainsi: "Il y a cent trente ans, les patriotes de Saint-Denis nous indiquaient le chemin de la dignité et de la liberté. Semence féconnde dont nous récoltons aujourd'hui les fruits." Il n'en faut pas davantage pour que le doute s'installe dans son esprit. Si je n'étais pas un partisan totalement convaincu du statu quo, pense-t-il, je considérerais que c'est "une grave atteinte à l'œuvre grandiose des La Vérendrye, sans compter que ce serait douter inconsidérément de la puissance d'expansion, de la vitalité inépuisable du Canada français tout entier". 
Libre à quiconque de rêver tout haut. La vérité ne change pas pour autant. L' “œuvre grandiose de La Vérendrye" n'appartient plus qu'à l'histoire et la "vitalité" du Canada français n'est pas "inépuisable". Les plaines de l'Ouest canadien ne sont pas plus taillées à "notre image et à notre ressemblance" que ne le sont les versants du Caucase. Un Français va-t-il revendiquer l'Espagne pour sa patrie en alléguant qu'un empereur, du nom de Charlemagne, y a un jour chevauché bannière au vent ?

Un lyrisme de mauvais aloi nous a distraits trop longtemps de la réalité. Ne retombons plus dans les mêmes ornières. Une culture et une langue ne sont pas des objets de luxe, mais des outils servant à gagner la vie de tous les jours. L'anémie et la mort culturelles guettent tout individu forcé à vivre et à travailler dans un climat qui lui est étranger. Personne ne doit vivre indéfiniment sur la brèche. La lutte, dis-je, est une phase passagère dans la vie de tout homme comme de toute nation. $\mathrm{Ni}$ l'un ni l'autre ne peuvent $y$ sacrifier toutes leurs forces vives sans risquer la décadence, voire l'extinction. Les sciences, les lettres, les arts, le commerce, l'industrie et l'agriculture réclament la totalité des énergies collectives.

Face à de tels impératifs, le Canada français - du moins l'image qu'on persiste à nous en faire - reste un mythe. A l'exception des Ontariens et des Acadiens vivant en bordure du sol québecois, les minorités francophones éparses sont vouées à une assimilation plus ou moins lente. Nul n'est à blâmer. La même loi universelle s'applique ici comme ailleurs.

Monsieur Gouin a grandement raison de dire que les combats se font désormais "à coups d'instruction, de progrès économiques, financiers et scientifiques". Mais comment mener efficacement la lutte sur ces nouveaux terrains sans l'intervention directe ou indirecte de l'Etat ? Et comment cette intervention pourra-t-elle nous servir si nous ne tenons pas en main les leviers de commande ? Dans le contexte moderne, l'autodétermination est le droit indispensable à l'existence des peuples. Le Québec ferait-il exception à la règle? 